\title{
Research Article \\ Electrical Power Analysis Using the Scattering Bond Graph
}

\author{
Roberto Tapia-Sánchez \\ División de Estudios de Posgrado, Facultad de Ingeniería Eléctrica, Universidad Michoacana de San Nicolás de Hidalgo, \\ 58000 Morelia, Mich, Mexico \\ Correspondence should be addressed to Roberto Tapia-Sánchez; rtsanchez@dep.fie.umich.mx
}

Received 6 September 2014; Accepted 6 January 2015

Academic Editor: Lucian Dascalescu

Copyright (c) 2015 Roberto Tapia-Sánchez. This is an open access article distributed under the Creative Commons Attribution License, which permits unrestricted use, distribution, and reproduction in any medium, provided the original work is properly cited.

The perspective of the well known concept of active and reactive power in electrical systems is addressed in this paper. This concept is presented from the point of view of the bond graph methodology. The scattering matrix concept is used in order to deal with these formulations. The concept of active and reactive power is presented with some examples derived from single-phase AC circuits. The formulations and simulations of these circuits are presented.

\section{Introduction}

Power concept is of central importance in electrical power systems. Reactive and active power concepts have been used since the beginning of the alternate current electrical generation. These power concepts can be encountered in the electric circuit theory, and they have been used naturally in the electrical power systems.

Reactive power $(Q)$ is defined to represent the quantity of electric power due to the load current that is not in phase with the source voltage. The average of this reactive power during one period of line frequency is zero. It means that this power does not contribute to energy transfer from the source to the load. Apparent power $(A)$ gives the idea of how much power can be delivered or consumed if the voltage and current are sinusoidal and perfect in phase. The power factor (PF) gives a relation between the average power actually delivered or consumed in a circuit and the apparent power at the same point. The higher the power factor, the better the circuit utilization. Consequently, the power factor is more efficient electrically as well as economically.

In this paper, the flow of power in an alternate current (AC) circuit is investigated. By using the concept of scattering and bond graph, the instantaneous power is resolved into two components. A plot of these components is obtained using the 20Sim software to observe that single-phase AC networks not only consume energy at an average rate but also borrow and return energy to its source. This leads to the basic definitions of average power and reactive power. The voltampere, which is a mathematical formulation based on the phasor forms of voltage and current, is introduced using the concepts of incident and reflected waves.

It is known that, through the bond graph methodology, any physical system can be modeled (i.e., mechanical, electrical, and thermodynamic) in the same reference frame. This methodology presents some properties that can be directly applied to the model [1]. A bond graph consists of subsystems linked together by half arrows, representing power bonds [2]. These bonds exchange instantaneous power at places called ports. The variables are forced to be identical when two ports are connected; the power variables are assumed to be functions of time. The different power variables are classified in a universal scheme and are called either effort $e(t)$ or flow $f(t)$. Their product $P(t)=e(t) \cdot f(t)$ is the instantaneous power flowing between the ports. As the instantaneous power is only presented in a bond, can it be possible to get the reactive power and apparent power, which are presented in an electrical power system? This is the question that this paper seeks to answer.

In order to answer this question, the relation between scattering matrix and bond graph is explored. Some publications have dealt with this formulation [3-6]. In the first publication [3], the concept of scattering matrix has been introduced by using the forewave and backwave concepts. 
The authors emphasize that wave scattering is an alternative approach to model physical systems. The property of the scattering operators and matrices, which deals with causal relations and conservation laws, has been indicated. In [4], the scattering matrix concept and the bond graph have been combined, and the constitutive relations of every single element have been addressed from the point of view of the scattering formalism. A bond graph transformation is proposed in order to formulate a reduced bond graph. This reduced bond graph manages two reduced power variables instead of the traditional effort and flow. From the reduced bond graph (transformed) both the transfer function and the scattering relation can be extracted. In [5], the name "bond graph scattering" appears as a result of the proposal, and the authors use the quadrupole concept in order to get the transfer function between two ports. Finally in [6], a more general view of the bond graph scattering is presented by adding the energy notion and their use in the study of linear and nonlinear, multienergy representation of physical systems.

In this paper, the bond graph scattering concept will be used in order to split up the instantaneous power into the two types of power ( $P$ and $Q$ ) commonly used when an electrical system is analyzed. Then, the power balance is demonstrated by means of examples focusing on the single-phase systems. Next, the transmission power between two voltage sources is considered, and the dependency of real power on voltage phase angle and of reactive power on voltage magnitude is established.

The outline of the paper is as follows: in the first part, the traditional electrical power is reviewed. The scattering bond graph is presented in Section 3. Section 4 describes some examples focusing on the single- phase circuits. Section 5 deals with a power flow study case. Finally, conclusions of the conducted investigation are drawn in Section 6.

\section{Review of Electrical Power}

In the AC circuits, energy storage elements (inductors and capacitances) may result in periodic reversals of the direction of energy flow. The averaged portion of power flow over a complete cycle resulting in a net transfer of energy in one direction is known as real or active power $(P)$. The portion of power flow due to stored energy, which returns to the source in each cycle, is known as reactive power $(Q)$.

The power theory based on active, reactive, and apparent power definition has been sufficient for the analysis and design of power systems. Nevertheless, some papers have been published showing that the conventional concept of reactive and apparent power loses its usefulness in nonsinusoidal cases [7]. Two approaches to power definitions under nonsinusoidal conditions were introduced in [8-10]. In $[8,9]$, the power was defined in the frequency domain, and in [10], it was defined in the time domain.

The introduction of power electronics technology introduced new conditions of power theory. This is because electronic power converters may bring out reactive power as well as harmonic current from power networks. Thus, the

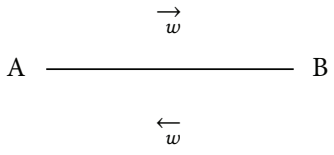

Figure 1: Bond between two elements.

conventional power theory based on average and RMS (root mean square) values [9] of voltages and sources cannot be applicable to the analysis and design of power converters and power networks.

The researches [11-13] presented the basic concepts to control the reactive power in order to compensate it.

In [14], the $p-q$ theory defines a set of instantaneous power in the time domain. This theory is focusing on a threephase circuit, and it always considers three-phase systems together, not as a superposition or sum of three single-phase circuits.

\section{Scattering Bond Graph (StBG)}

This section presents a recapitulation of the scattering bond graph and also gives a more general standpoint of its use.

As mentioned in [3], system description requires knowing its elements and interconnections. In order to model any physical system, it is necessary to define the system boundary. In case of a boundary breaking up the system into two subsystems, the interaction between subsystems can be described by a duplex pair of oppositely directed signals. Whatever bond exists between two parts of a system may be equivalently represented as a pair of directed signals.

The forewave $(\vec{w})$ and backwave $(\overleftarrow{w})$ have been addressed in [3]. Two systems A and B are joined by a single line, which represents the interaction of the two waves. There is a power exchange between the two systems; part of the complete power could be moving from $A$ to $B$ and part from $B$ to $A$. Figure 1 shows this interaction between the two systems.

If $P$ is defined as the net power flowing from $\mathrm{A}$ to $\mathrm{B}$, then $\vec{P}$ is defined as the fore-power and $\widetilde{P}$ as the backpower. Each of these bilateral power flows represents the integration at the port of a scattering flux, which is defined as the product of local scattering density and local velocity of energy propagation. As the forewave and backwave have units of root power, they can be represented using a single power bond.

The net power flow is considered positive in the foredirection. Then, the net power can be expressed as a function of the waves as follows:

$$
\begin{aligned}
P & =\vec{P}-\overleftarrow{P}=\frac{\vec{w}^{2}}{2}-\frac{\overleftarrow{w}^{2}}{2} \\
& =\frac{1}{\sqrt{2}}(\vec{w}+\overleftarrow{w}) \cdot \frac{1}{\sqrt{2}}(\vec{w}-\overleftarrow{w})=e \cdot f .
\end{aligned}
$$

Equation (1) shows the relationship between the scattering variables and the normalized effort and flow variables.

In this paper is proposed the graphical representation of the scattering bond as shown in Figure 2. 


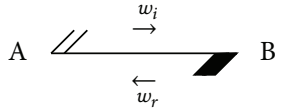

FIGURE 2: Scattering bond.

TABLE 1: Scattering source equations.

\begin{tabular}{|c|c|c|}
\hline & Effort source & Flow source \\
\hline 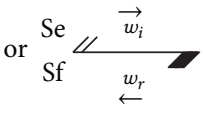 & $w_{i}=\sqrt{2} \cdot V-w_{r}$ & $w_{i}=\sqrt{2} \cdot I+w_{r}$ \\
\hline $\mathbb{Z}_{\stackrel{w_{r}}{\leftarrow}}^{\vec{w}_{i}}{ }_{\mathrm{Sf}}^{\mathrm{Se}}$ or & $w_{r}=\sqrt{2} \cdot V-w_{i}$ & $w_{r}=-\sqrt{2} \cdot I+w_{i}$ \\
\hline
\end{tabular}

The forewave and backwave are called incident $\left(w_{i}\right)$ and reflected $\left(w_{r}\right)$ waves, respectively (as it was made in [4]). The bold half-arrow indicates the sense of the incident wave, while the double half-arrow the sense of the reflected wave. The junction, ports, and elements have the same representation as in a traditional bond graph; only their mathematical relations change. Figure 3 shows the basic elements used in order to formulate a StBG.

The source elements (Se and Sf) give their variable ( $V$ for voltage and $I$ for the current) via the incident wave. The original values are normalized by the operator $\sqrt{ } 2$ as is common in the scattering representation $[15,16]$. These sources can be visualized from an energetic conservation point of view [6], by using the relationship given in (1). Then, the sources of effort and flow can be interpreted as four scattering sources. Table 1 summarizes these sources.

The relations shown in Table 1 make references to an electrical circuit because voltage $(V)$ and current $(I)$ variables have been considered as the effort and flow, respectively. It is important to note that the combination of these four sources arises because the incident wave defines the sign convention.

The scattering variables $\left(w_{i}, w_{r}\right)$ are defined at each port of the system and always adopt the positive power convection in a system.

As shown in Figure 3, the constitutive relation for $C$, $I$, and $R$ elements is normalized by a resistance $R_{0}$. The normalization by $R_{0}$ corresponds to an internal resistance or impedance considered in a system (i.e., source, resistance, and capacitance). This resistance makes it possible to quantify the power transferred between two systems.

By considering a linear capacitance $C$, the relation between $w_{i}$ and $w_{r}$ is given by $w_{i} / w_{r}=Z / R_{0}$, where $R_{0}$ is the normalized resistance and $Z$ is the impedance of the element: $Z=1 / C s$ ( $s$ being the Laplace operator). From this impedance and the scattering relation $\left[w_{r}\right]=S\left[w_{i}\right]$ given between two ports, it is simple to find the relation shown in Figure 3 by replacing the $s$ operator by $\rho$. The constitutive relation of this element contains a zero in the right half-plane and is nonminimum phase element, although, clearly, it is normally considered to be physically real. The compliance element is obtained in the same manner.
The scattering relation for a one-port resistor has the extreme values of -1 and +1 when $R>R_{0}, S_{R}<0$ and when $R<R_{0}, S_{R}>0$, respectively. Then, when $R=R_{0}, S_{R}$ is zero in both cases and the resistor is said to be perfectly matched [3, 17-19].

This paper is focusing on single-phase AC circuits; nevertheless, the StBG can be used in all physical systems including those that do not have alternate input sources.

\section{Single-Phase AC Circuits}

Electrical power systems commonly use an impedance load connected to a source so that the analysis can be simplified by using the phasor concept. This concept focuses on complex values having a magnitude and a phase in order to calculate the power using those vector quantities.

In the bond graph methodology, the impedance element has already been formulated [19]. In this publication, another element (besides the nine basic elements) is proposed as the impedance element, namely, $Z$-element. With the use of this $Z$-element, the notion of the interaction between different elements is lost because two or more basic elements can be grouped into one.

Next is proposed the use of the scattering bond graph in order to keep close to the structure of the analyzed circuit. Later, two basic examples of a single-phase electrical system are presented.

4.1. Circuit VR. The basic AC electrical circuit is composed only of a resistive load $(R)$ connected in parallel to the alternative voltage source $(V)$. Figure 4 shows the StBG of the $V R$ circuit.

As shown in Figure 4, the two variables in each scattering bond are the incident and the reflected waves $\left(w_{i}, w_{r}\right)$. The mathematical formulation is made by considering the relations given earlier and is as follows.

The source MSe and the $R$ element have the mathematical relations given by (2) and (3), respectively.

Consider

$$
\begin{aligned}
& w_{i 1}=\sqrt{2} v-w_{r 1}, \\
& w_{r 2}=S w_{i 2}=\frac{r-1}{r+1} w_{i 2},
\end{aligned}
$$

where $r=R / R_{0}$. As the two components are joined by a 1 junction, the scattering relation corresponds to

$$
S=\left[\begin{array}{cc}
0 & -1 \\
-1 & 0
\end{array}\right] .
$$

Then, considering these relations, the incident and reflected waves in each scattering bond are given by

$$
\begin{aligned}
& w_{r 2}=-\left(\frac{r-1}{2 r}\right) \sqrt{2} v, \\
& w_{i 2}=-\left(\frac{r+1}{2 r}\right) \sqrt{2} v,
\end{aligned}
$$



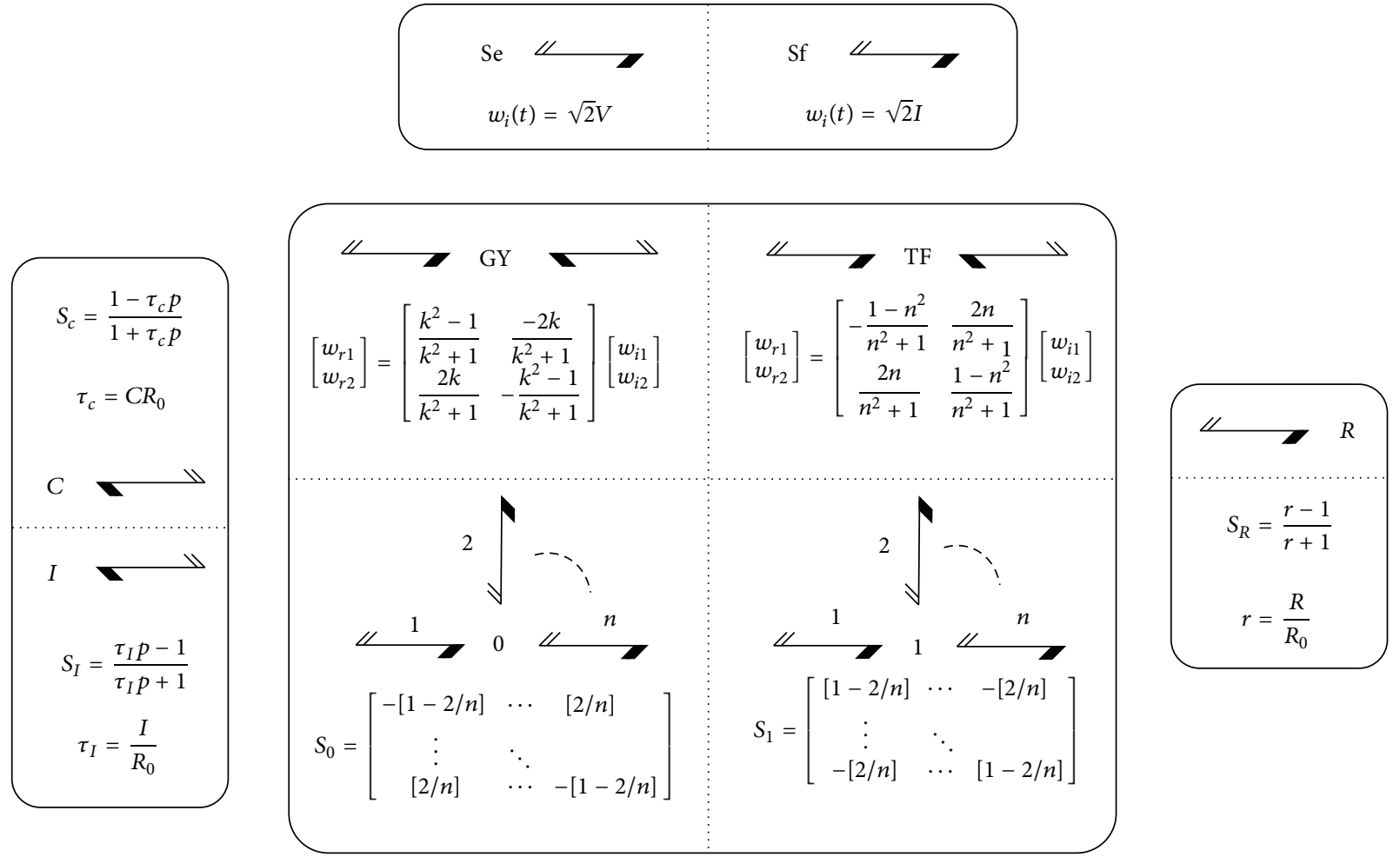

Figure 3: Scattering bond graph relations.

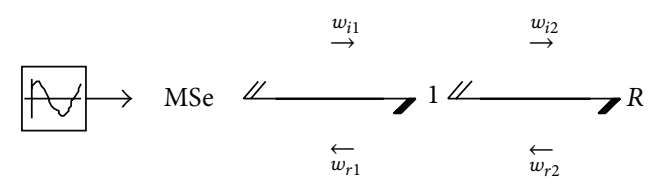

Figure 4: StBG of the VR circuit.

$$
\begin{aligned}
& w_{r 1}=\left(\frac{r+1}{2 r}\right) \sqrt{2} v, \\
& w_{i 1}=\left(\frac{r-1}{2 r}\right) \sqrt{2} v .
\end{aligned}
$$

Equations (5) and (6) represent the scattering waves of the StBG of Figure 4; they do not match the traditional effort and flow present in a bond graph. In any case, the original variables of effort and flow can be recovered [6] from the new variables using (1).

The objective to use the scattering variables is to obtain the active and reactive power directly by regarding the incident and reflected waves. Then, the power delivered by each element is given by

$$
P_{t}=P_{i}-P_{r}=\frac{\left(w_{i}\right)^{2}}{2}-\frac{\left(w_{r}\right)^{2}}{2}
$$

where $P_{t}$ is the total power and $P_{i}$ and $P_{r}$ are the incident and reflected power, respectively. By substituting (5) in (7), the total power dissipated by the $R$ element is calculated as follows:

$$
P_{t}=\frac{(-((r+1) / 2 r) \sqrt{2} V)^{2}}{2}-\frac{(-((r-1) / 2 r) \sqrt{2} V)^{2}}{2}=\frac{V^{2}}{r}
$$

Equation (8) agrees with the usual power expressed in a resistive element.

In order to simulate the StBG of Figure 4, a library for the 20Sim software has been developed. The parameters used for the simulation are source $v=100 * \sin (2 \pi 50)$ and $R=$ $1.25 \Omega$. Figure 5 shows the incident and reflected waves in each scattering bond.

The responses $w_{i 1}$ and $w_{r 1}$ correspond to the source MSe and $w_{i 2}$ and $w_{r 2}$ to the resistive $R$-element. It is shown that responses are the same for the two elements from the point of view of magnitude peak of the sinusoidal curves.

The source incident wave has a maximum value of 127.27, while for the source reflected wave it is 14.14. The same is the case for the scattering variables of the $R$-element. The values obtained by a traditional analysis do not match these values; hence, the scattering variables are used.

The responses in the conventional variables $(e=$ voltage and $f=$ current) for the MSe source are presented in Figure 6.

The effort and flow responses match exactly the expected values for the voltage $\left(V_{\max }=100 \mathrm{~V}\right)$ and current $\left(I_{\max }=\right.$ $80 \mathrm{~A})$ if a traditional bond graph is used. 

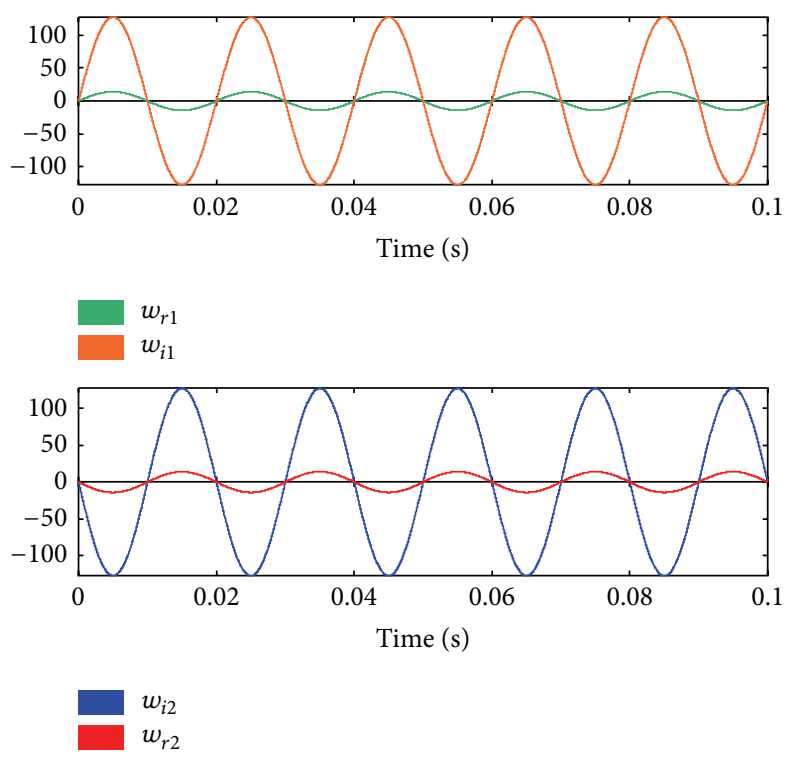

FIgURE 5: Reflected and incident waves for the $V R$ circuit.

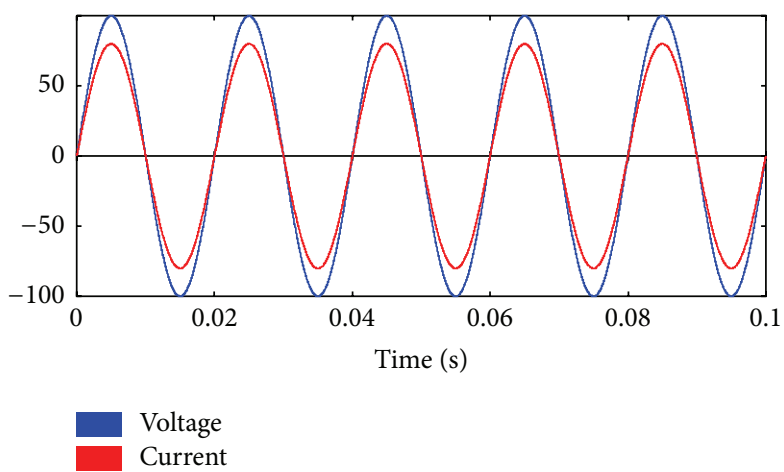

FIGURE 6: Effort and flow variables responses.

As mentioned earlier, the objective to use the scattering variables is to deal with the active and reactive power directly in the StBG. Figure 7 shows the power responses for the $R$ element.

The instantaneous power is presented in Figure 7. As expected, the frequency of the instantaneous power is twice the frequency of the input source. The reflected power $P_{r R}$ is smallest compared with the incident power $P_{i R}$. Actually, the total power is slightly lower than the incident power because the reflected power is subtracted.

Generally, the average value of the instantaneous power is considered (Figure 8) as the power delivered or absorbed by each element.

In conclusion, it is observed that the incident power is slightly higher than the total power and the reflected power has a very small value.

From this result, it can be concluded that the incident power has a correspondence with the active power in an electrical circuit, the reflected power has a correspondence with the reactive power, and the apparent power can be related to the total power.
It is important to note that the $V R$ circuit does not have a dynamic element; thus, only active power is present in the circuit. Nevertheless, in the example presented earlier, incident (active) power, reflected (reactive) power, and total (apparent) power are present. This discrepancy can be interpreted on the basis of bond graph methodology as follows: to any action (effort) there is a reaction (flow); this is the principle of causality. Extrapolating this concept to the scattering variables, for any inflow wave, there is an outflow wave.

Despite the presence of reflected power in the StBG, its contribution is minimum and is compensated with the slightly higher value of the incident power. Actually, the difference is the same: reflected power is equal to 50 and the slight value of incident power is equal to 50 . Their sum gives the total power (apparent) in the element.

4.2. Circuit VRL. Circuit VRL is composed of a voltage source, a resistance, and an inductor, all connected in series. Figure 9 shows the StBG of this circuit. 

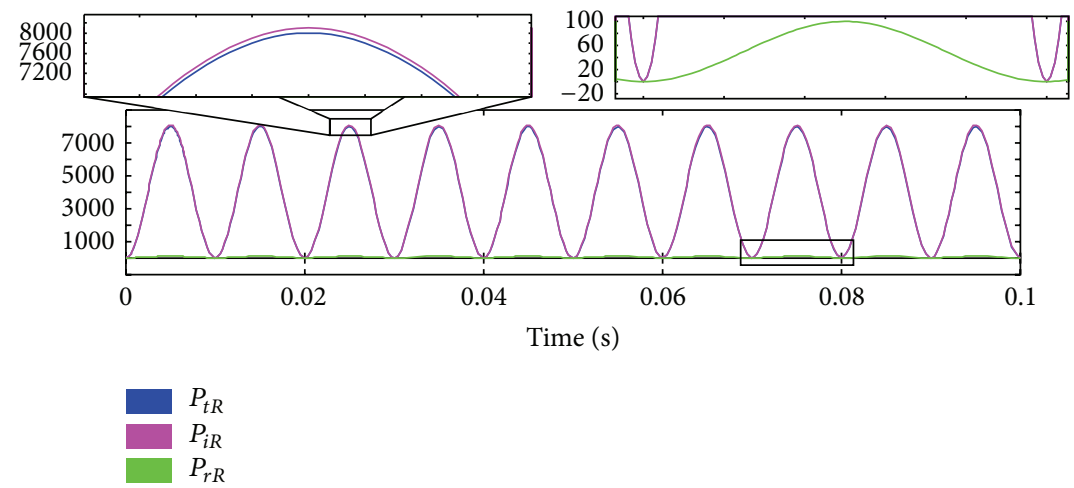

Figure 7: Power waves responses for the R-element.

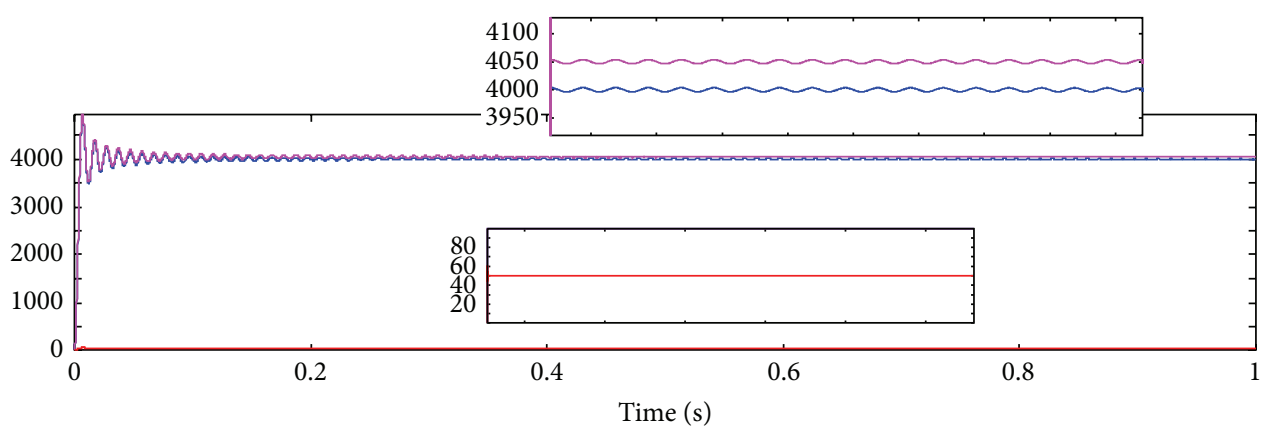

$P_{t}$-average

$P_{i-\text { average }}$

$P_{r}$ average

FIgURE 8: Average power for the $V R$ circuit.

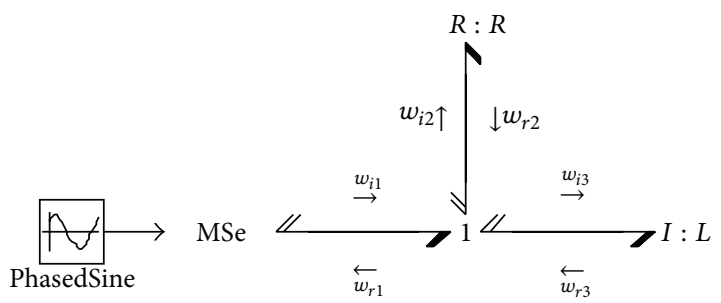

FIGURE 9: StBG of a VRL electrical circuit.

There is a dynamical element in this StBG, so a voltage and current phase shift will be present.

As in the case of circuit $V R$, the StBG of Figure 9 is simulated. Figure 10 shows the scattering waves' responses. The numerical values for the simulation are $R=0.625 \Omega$, $L=0.0034457 \mathrm{H}$, and $V=100 * \sin (2 \pi 50)$.

Incident and reflected waves in each element have different amplitudes and their own phases. It is important to note that $R$ and $L$ incident waves $\left(w_{i R}, w_{i L}\right)$ have a phase shift around $60^{\circ}$. This phase shift is actually the value that can be obtained mathematically and corresponds to the angle of the impedance load $\left(z=1.25,60^{\circ}\right)$. The same phase shift is visualized between the $w_{r R}$ and $w_{r L}$.

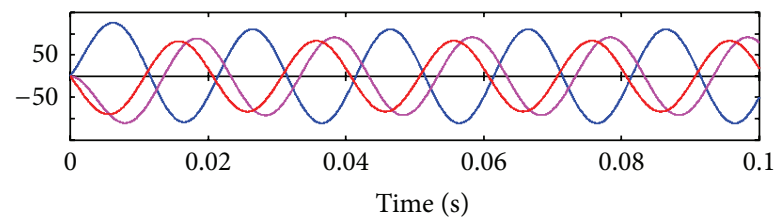

$w_{i s}$
$w_{i R}$
$w_{i L}$
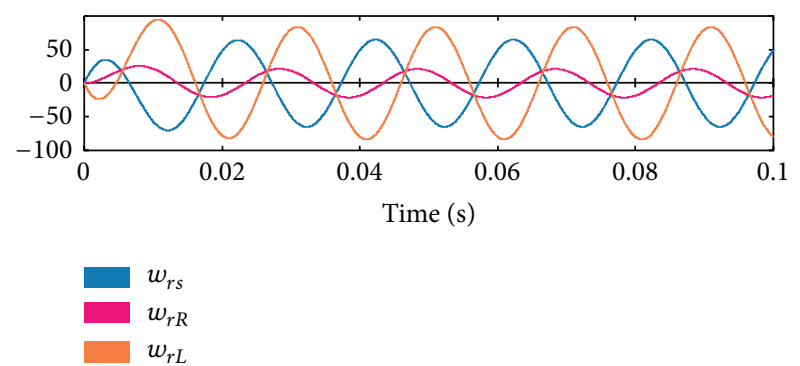

FIGURE 10: Scattering wave's responses of the VRL circuit.

The efforts and flows can be obtained from the final results. Figure 11 shows the power presented in each wave.

As in the case of $V R$ circuit, the instantaneous curves of power are obtained. It is observed that the source presents 


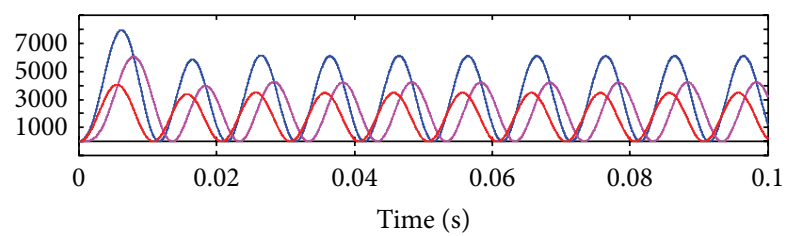

$P_{i s}$

$P_{i R}$

$P_{i L}$

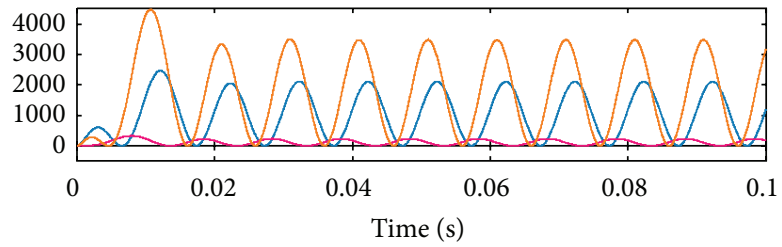

$P_{r s}$
$P_{r R}$
$P_{r L}$

FIGURE 11: Power waves responses in the $V R L$ circuit.
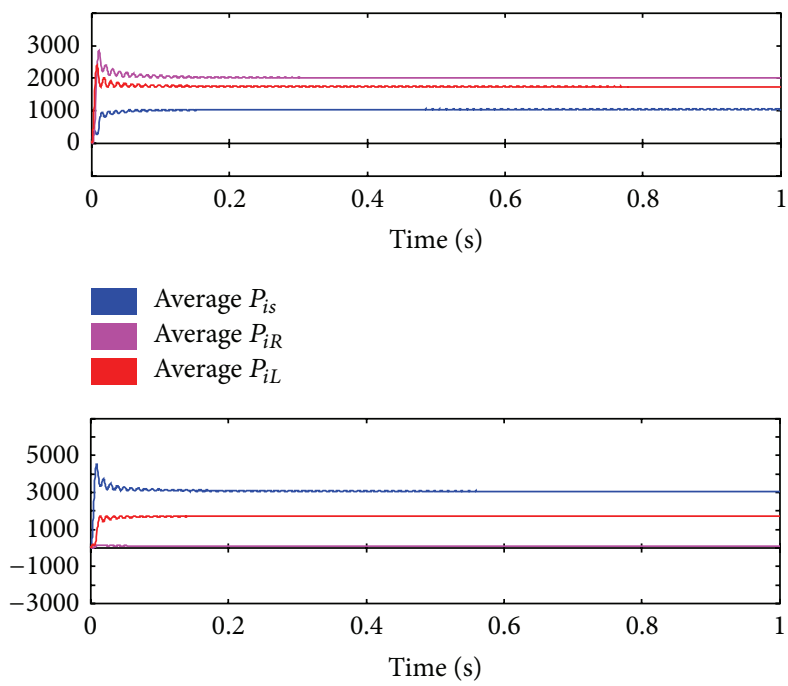

Average $P_{r s}$

Average $P_{r R}$

Average $P_{r L}$

FIgURE 12: Average power responses for the VRL circuit.

the larger amplitude when dealing with incident power $\left(P_{i s}\right)$. However, when dealing with reflected power, the larger amplitude is presented by the inductor element $\left(P_{r L}\right)$. This is normal for this circuit because the reactive power is delivered by the inductor.

If the total power in each element is calculated, from Figure 12 it is found that $P_{t R}=1890, P_{t L}=6$, and $P_{t s}=$ -2009 , which correspond to the total power in the $R$ element, the $L$ element, and the source, respectively. Some interesting observations can be made with these results.

(i) The fact that the total power in the $L$ element is close to zero is because this element does not contribute any usable power. Actually, this element contributes only reflected power. In the language of electrical systems, it means that the inductor contributes only with reactive power.

(ii) The total power in the $R$ element and the total power in the source have a contrary sign, and they are almost the same.

(iii) There must be a balance in the power delivered or absorbed for each element. If all the total power is added up, it can be shown that it is not balanced. Nevertheless, there is a balance in power, but, for showing this balance, all the power needs to be taken into consideration (incident power and reflected power). In this case, $P_{t R}+P_{t L}+P_{t s}=-113$, which is equal to the value of $P_{r R}$ (with a positive sign). With this result, is verified the same behavior as presented in the $V R$ circuit (Figure 4).

The active and reactive power values can be obtained by regarding the total power waves in the sinusoidal reference, as shown in Figure 13.

In the $R$ element, the total power has a maximum value of 4000. Active power is obtained by taking its mean value. The maximum value of the total power in the $L$ element is 3450 , which corresponds directly to the reactive power value in the circuit.

Thus, it can be concluded that, for a given StBG, the mean total power waves in the $R$ elements correspond to the electrical active power, and the total power waves in the $L$ or $C$ elements correspond to the electrical reactive power.

Other circuit configurations, that is, capacitance and resistance in parallel with a source of current (IRC), VRLC, or a mix of different basic circuits using a single-phase electrical system, can be done simply by using the basic StBG elements.

\section{Power Flow in a Single-Phase System}

Power flow is an important concept in electrical power systems. This concept is used in transmission, distribution and also when the power electronic converters are connected into the electrical network.

Here, the basic concept of power flow in a single-phase electrical system is considered.

Consider two ideal voltage sources connected by a transmission line as shown in Figure 14.

It is known that, in this circuit, small changes in phase shift between the two sources will have a significant effect on the real power flow, while small changes in the voltages' amplitudes will not have an appreciable effect on it. Therefore, the flow of real power in a transmission line is governed by the angle difference between both sources. For maintaining transient stability, the power system is usually operated with small angle difference. Moreover, the reactive power flow is determined by the magnitude difference of terminal voltages.

The traditional analysis (using the phasor concept) of this circuit is given here by considering the numerical values $V_{1}=$ $120 \angle-5^{\circ} ; V_{2}=100 \angle 0^{\circ} ;$ impedance line $z=1+j 7 \Omega$. Then, 

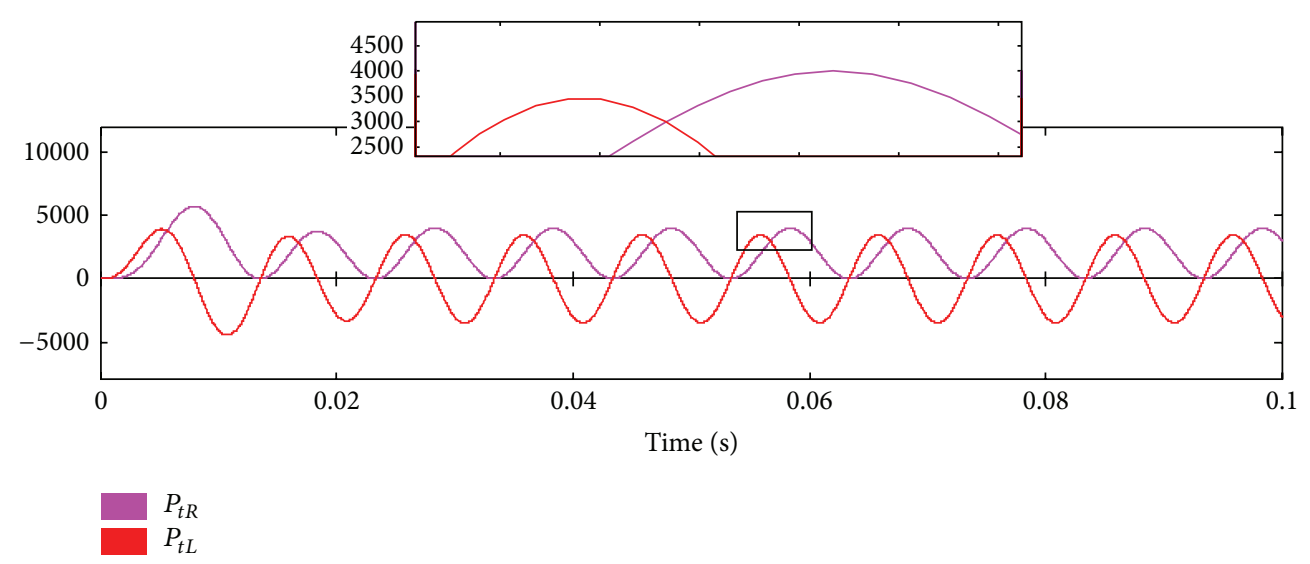

FIgURe 13: Power waves in the VRL StBG.

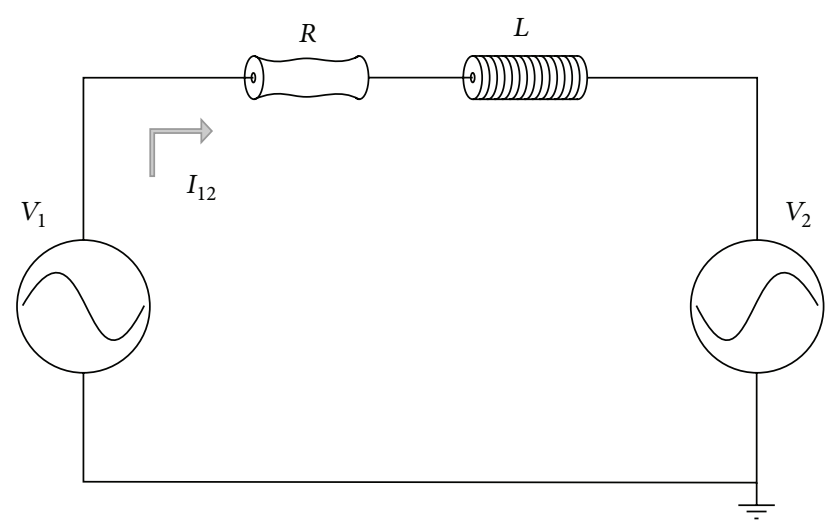

FIgURE 14: Two interconnected voltages sources.

the circulating current from source $1\left(V_{1}\right)$ to source $2\left(V_{2}\right)$ is given by $I_{12}$, and $I_{21}$ is the reverse current. Consider

$$
\begin{aligned}
& I_{12}=\frac{120 \angle-5^{\circ}-100 \angle 0^{\circ}}{1+j 7}=3.135 \angle-110.02^{\circ}, \\
& I_{21}=\frac{100 \angle 0^{\circ}-120 \angle-5^{\circ}}{1+j 7}=3.135 \angle 69.98^{\circ} .
\end{aligned}
$$

The apparent power delivered or absorbed by each source is given by

$$
A_{12}=V_{1} I_{12}^{*}=376.2 \angle 105.02^{\circ}=-97.5 \mathrm{~W}+j 363.3 \mathrm{VAR} \text {, }
$$

$$
A_{21}=V_{2} I_{21}^{*}=313.5 \angle-69.98^{\circ}=107.3 \mathrm{~W}-j 294.5 \mathrm{VAR} .
$$

Equation (10) means that source 1 absorbs $97.5 \mathrm{~W}$ of active power and delivers 363.3 VAR. From (11), we can see that the source 2 provides $107.3 \mathrm{~W}$ and absorbs 294.5 VAR.

Also, the loss in the transmission line can be calculated by

$$
A_{L}=A_{12}+A_{21}=9.8 \mathrm{~W}+j 68.8 \mathrm{VAR} .
$$

The corresponding StBG for the interconnected voltage sources is shown in Figure 15.
The StBG presented in Figure 15 has the same structure (as the examples presented earlier), but it can be seen that the scattering bonds 4 and 5 are added. The scattering bond 4 is kept intentionally taking the convention current into consideration. This means that the power is transferred from source $V_{1}$ to source $V_{2}$.

The parameters taken in the simulation are the same as those taken in the electrical system of Figure 14: MSe: $V_{1}=$ $120 \sin \left(\omega t-5^{\circ}\right)$; MSe: $V_{2}=100 \sin (\omega t) ; R=1 \Omega$ and $L=22.28 \mathrm{H}$; where $\omega$ is the angular frequency $\omega=2 \pi 50$. Figure 16 shows the scattering variables in each bond.

The incident and reflected waves can been interpreted as follows.

In case of $w_{i} s_{1}$ and $w_{i} s_{2}$, a phase shift of $-5^{\circ}$ is visualized (although they have $180^{\circ}$ phase shift) and the amplitude difference between these two sources is also considered. For the $w_{r R}$, which is the reflected wave in the $R$ element, it can be seen that its value is zero. This is due to the chosen $R$ element value, which is equal to $1 \Omega$; then, the scattering relation inside this element is $S=0$ (the element is matched).

The incident power and reflected power in the sources are given in Figure 17.

From Figure 17, the average total power in the source 1 is -96.5 , while it is 106.5 for the source 2 . These two values 


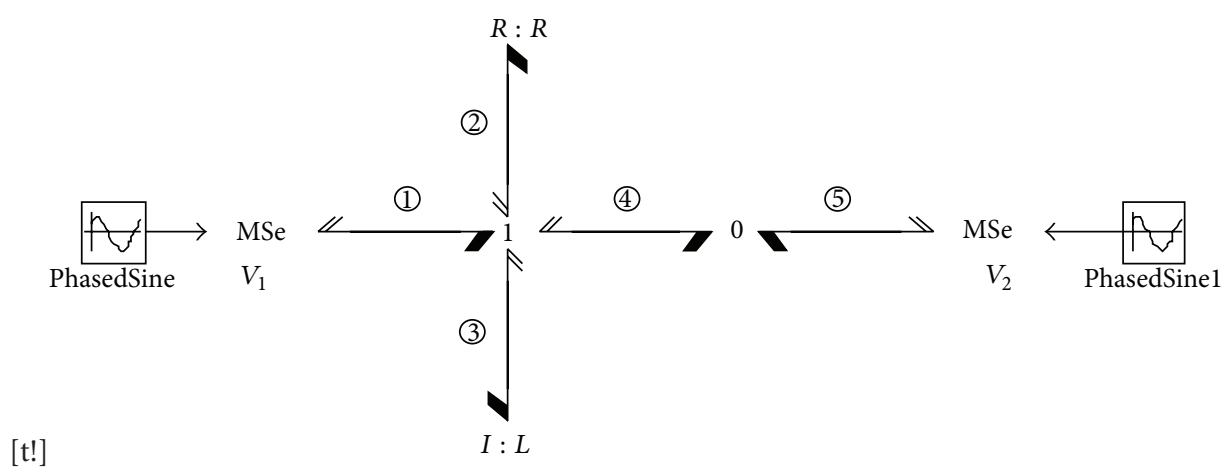

Figure 15: StBG of two interconnected sources.
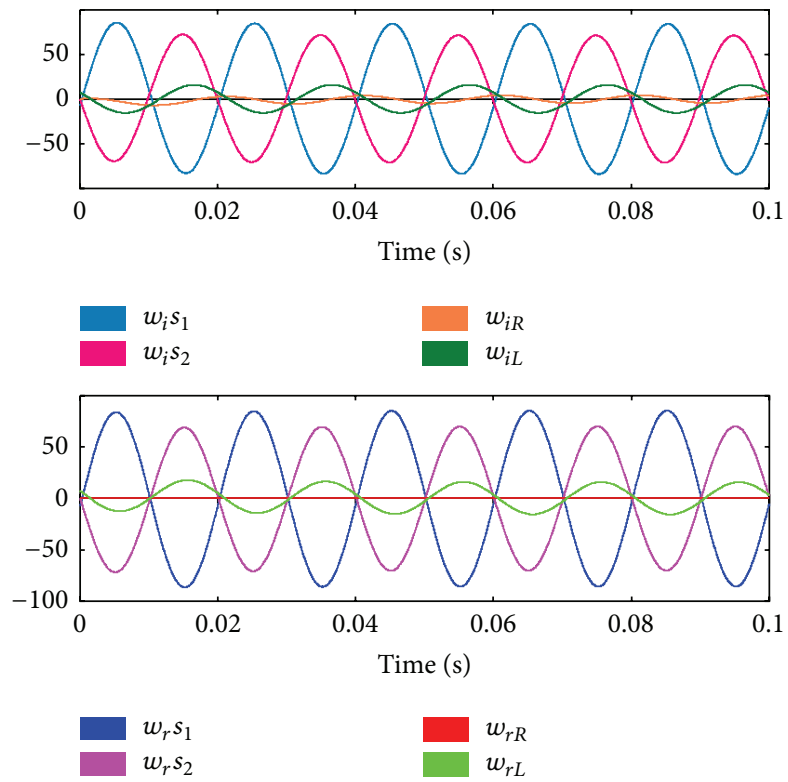

FIGURE 16: Power waves for the StBG of Figure 15.

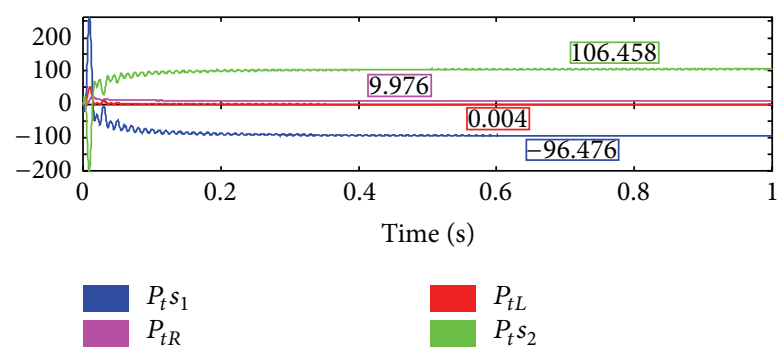

FIGURE 17: Sources average power responses.

match closely the active power values calculated earlier ((10) and (11)).

When the power flow is analyzed, it is important to know the active power and reactive power delivered or absorbed by each source. The two sources are not dynamic elements; however, they can deliver or absorb reactive power. In order

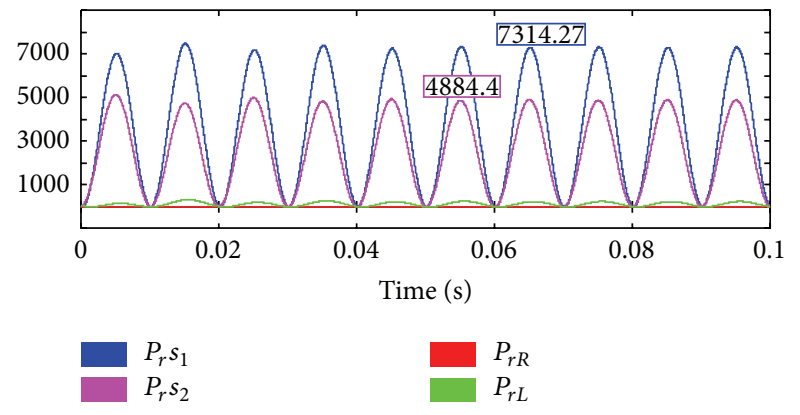

FIGURE 18: Reflected waves in the StBG of Figure 15.

to get the values of this reactive power it is necessary to see the response of the reflective power waves in these elements. Figure 18 shows the reflected power waves.

As earlier, the reactive power value is taken directly from the peak value of the reflected power waves. For this case, it can be seen that the peak value of the reflected power waves for sources $V_{1}$ and $V_{2}$ is $P_{r} s_{1}=7314.27 \mathrm{VAR}$ and $P_{r} s_{2}=$ 4884.4 VAR, respectively. These values do not match (10) and (11); nevertheless, this can be interpreted as follows: the two sources have a difference of 20 volts in their amplitudes, and as mentioned before, the difference between two sources has a direct impact on the reactive power interchanged between them. Then, if the peak value of these two reflected waves is divided by this difference ( 20 volts), the reactive power can be obtained $\left(P_{r} s_{1}=365.7 \mathrm{VAR}\right.$ and $\left.P_{r} s_{2}=244.2 \mathrm{VAR}\right)$.

It is evident that, in this part, the absorbed or generated reactive power in each source cannot be differentiated. It means that the sign cannot be obtained directly. In order to solve this disadvantage, it is necessary to look at the phase shift between these two sources and then assign the corresponding sign.

The power losses in the elements $R$ and $L$ are shown in Figure 19.

Same as before, the active power $(P=10 \mathrm{~W})$ is obtained by taking the mean value of the total power wave in the $R$ element. The maximum value of the total power in the $L$ element is $69.9 \mathrm{VAR}$, which corresponds directly to the reactive power loss in the transmission line. 


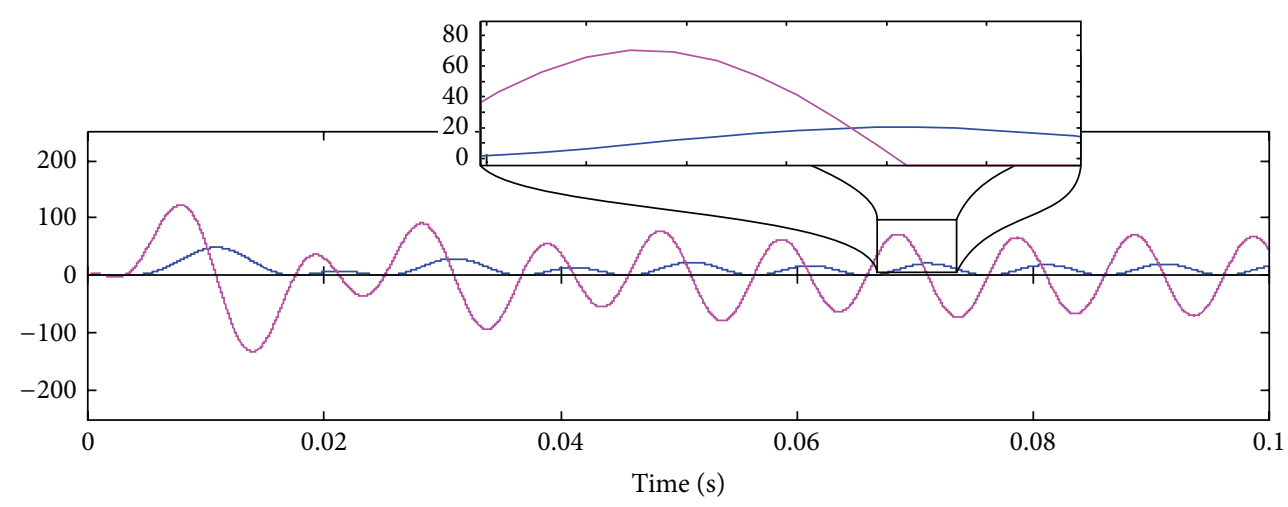

FIGURE 19: Losses power in the StBG of Figure 15.

\section{Conclusions and Future Work}

The active power and reactive power were obtained from the scattering bond graph. The problem of visualizing the reactive power in a bond graph was resolved. A library developed in the software 20Sim allowed simulating the single-phase electrical circuits.

The exposed scattering bond graph analysis represents an alternative to the classical phasor analysis. This has been shown through different examples.

The use of the StBG allowed obtaining an online reactive and active power calculation, when the system is simulated. As the proposed analysis is based on a graphical methodology, the power interaction can be easily shown in a system. Besides, the different techniques already developed by the traditional bond graph methodology can be applied to this StBG formulation.

The scattering variables have allowed obtaining the active and reactive power directly by regarding the incident and reflected waves. This means that the system has been treated in a different reference frame (power frame) in which the systems interaction was described by a duplex pair of oppositely directed signals. Since different variables have been used, the traditional variables (i.e., voltage, current) can be obtained by using the scattering and normalized relationship.

In the next study, the balanced and unbalanced threephase circuits will be presented using the concept exposed here. Also, the use of incident and reflected variables is visualized in order to derive the control laws necessary in the electrical systems. It is important to note that the StBG does not apply exclusively to electrical systems; it can also be applied to other systems where it could be necessary to interpret the results.

\section{Conflict of Interests}

The author declares that there is no conflict of interests regarding the publication of this paper.

\section{References}

[1] G. Dauphin-Tanguy, Les Bond Graphs, Hermès Science, 2000.

[2] D. Karnopp, D. Margolis, and R. Rosenberg, System Dynamics: A Unified Approach, John Wiley \& Sons, New York, NY, USA, 1990.

[3] H. M. Paynter and I. J. Busch-Vishniac, "Wave-scattering approaches to conservation and causality," Journal of the Franklin Institute, vol. 325, no. 3, pp. 295-313, 1988.

[4] M. Amara and S. Scavarda, "A procedure to match bond graph and scattering formalisms," Journal of the Franklin Institute, vol. 328, no. 5-6, pp. 887-899, 1991.

[5] A. Kamel and G. Dauphin-Tanguy, "Power transfer in physical systems using the scattering bond graph and a parametric identification," Systems Analysis Modelling Simulation, vol. 27, pp. 1-13, 1996.

[6] R. G. Longoria, "Wave-scattering formalisms for multiport energetic systems," Journal of the Franklin Institute, vol. 333, no. 4, pp. 539-564, 1996.

[7] W. V. Lyon, "Reactive power and unbalanced circuits," Electrical World, vol. 75, no. 25, pp. 1417-1420, 1920.

[8] C. I. Budeanu, Puissances réactives et fictives, Institut Romain de l'Energie, Bucarest, Romania, 1927.

[9] C. I. Budeanu, The Different Options and Conceptions Regarding Active Power in Non-Sinusoidal Systems, vol. 4, Institute Romain de l'Energie, 1927.

[10] S. Fryze, "Wirk-, Blind- und Scheinteistung in elektrischen Stromkreisen mit nicht-sinusformigen Verlauf von Strom und Spannung," Przegląd Elektrotechniczny (Polish), no. 7, pp. 193203, no. 8, pp. 225-234, no. 22, 673-676, ETZ, no. 25-29, 1931 (German).

[11] H. Sasaki and T. Machida, "A New method to eliminate AC harmonic currents by magnetic flux compensation-considerations on basic design," IEEE Applications on Power Apparatus and Systems, vol. 90, no. 5, pp. 2009-2019, 1971.

[12] M. S. Erlicki and A. Emanuel-Eigeles, "New aspects of power factor improvement part I-theoretical basis," IEEE Transactions on Industry and General Applications, vol. 4, no. 4, pp. 441446, 1968. 
[13] T. Fukao, H. Lida, and S. Miyairi, "Improvements of the power factor of distorted waveform by thyristor based switching filter," Transaction of the IEE-Japan Part B, vol. 92, no. 6, pp. 342-349, 1972.

[14] H. Akagi, Y. Kanazawa, and A. Nabae, "Instantaneous reactive power compensator comprising switching devices without energy storage components," IEEE Transactions on Industry Applications, vol. 20, no. 3, pp. 625-630, 1984.

[15] H. J. Carlin and A. B. Giordano, Network Theory: An Introduction to Reciprocal and Nonreciprocal Circuits, Prentice-Hall, Englewood Cliffs, NJ, USA, 1964.

[16] R. W. Newcomb, Linear Multiport Synthesis, McGraw-Hill, New York, NY, USA, 1966.

[17] K. Kurokawa, "Power waves and scattering matrix," IEEE Transactions on Microwave Theory and Techniques, vol. 13, no. 2, pp. 194-202, 1965.

[18] H. J. Carlin and A. B. Giordano, Networks Theory: An Introduction to Reciprocal and Non-reciprocal Circuits, Prentice Hall, Englewood Cliffs, NJ, USA, 1964.

[19] K. Kurokawa, An Introduction to the Theory of Microwave Circuits, Academic Press, New York, NY, USA, 1969. 

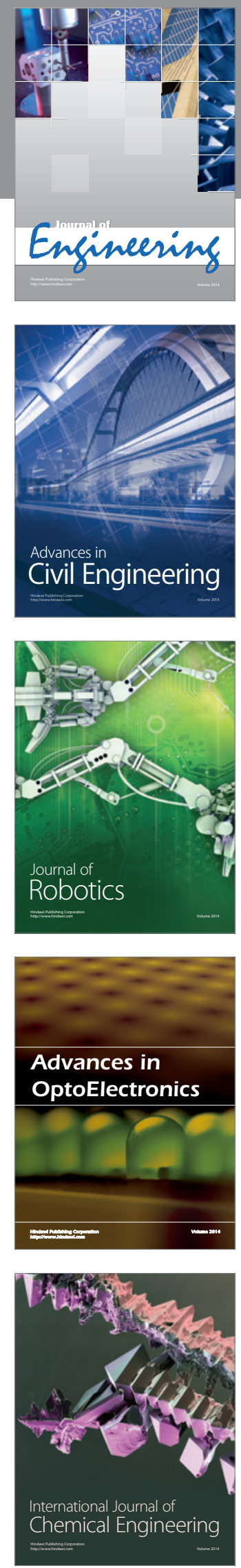

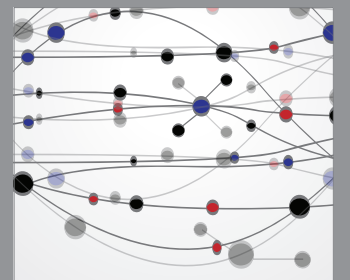

The Scientific World Journal
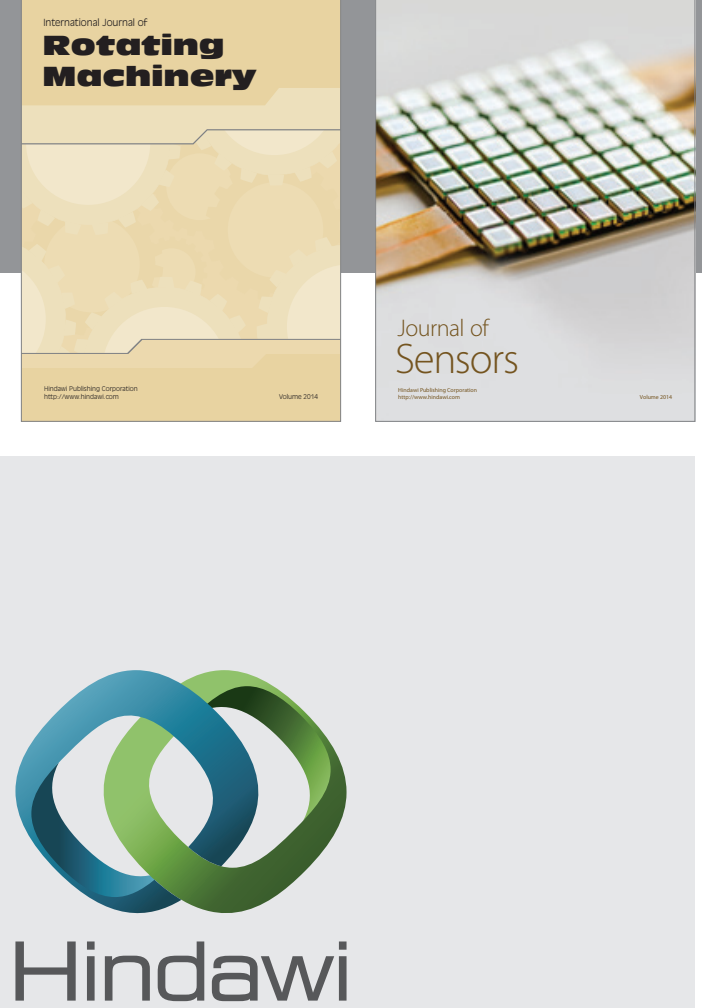

Submit your manuscripts at http://www.hindawi.com
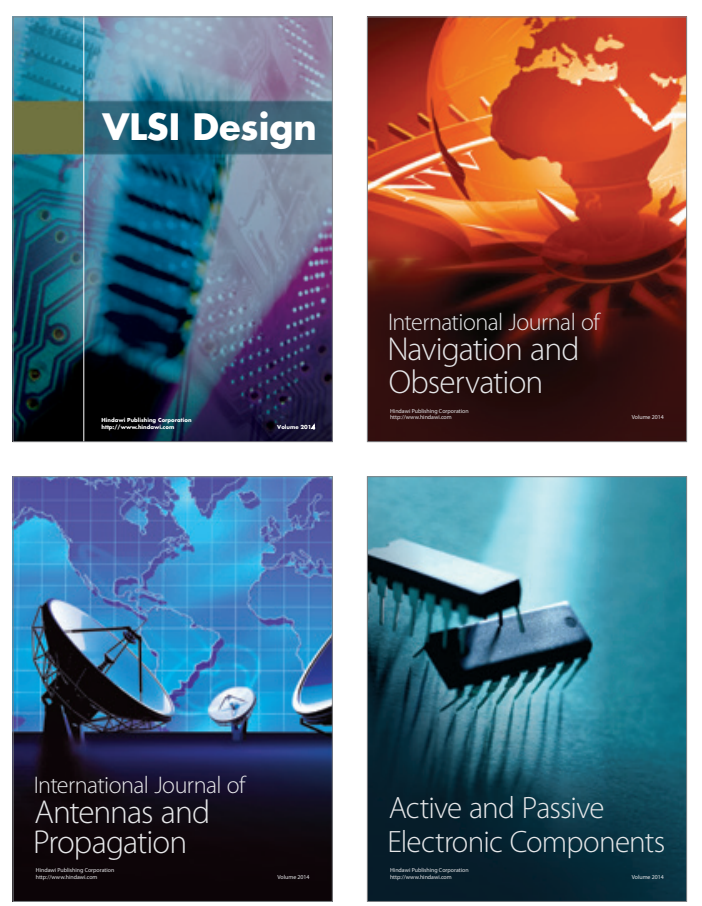
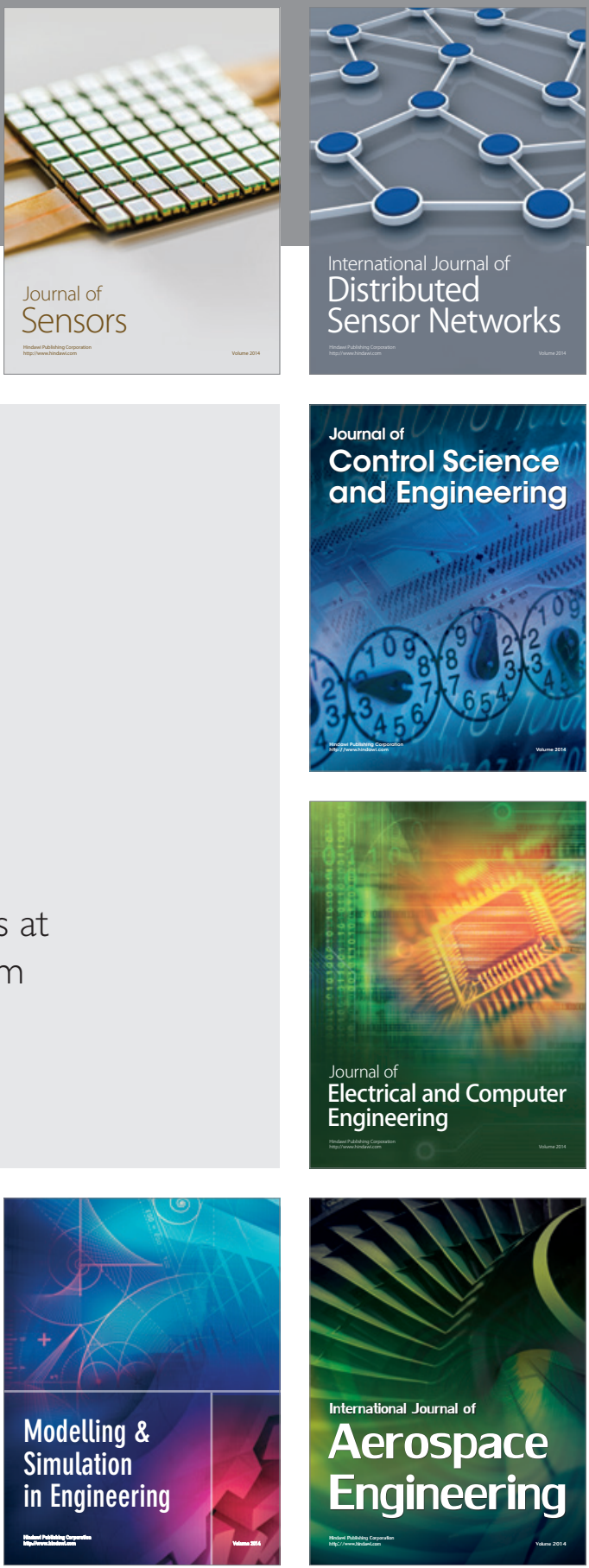

Journal of

Control Science

and Engineering
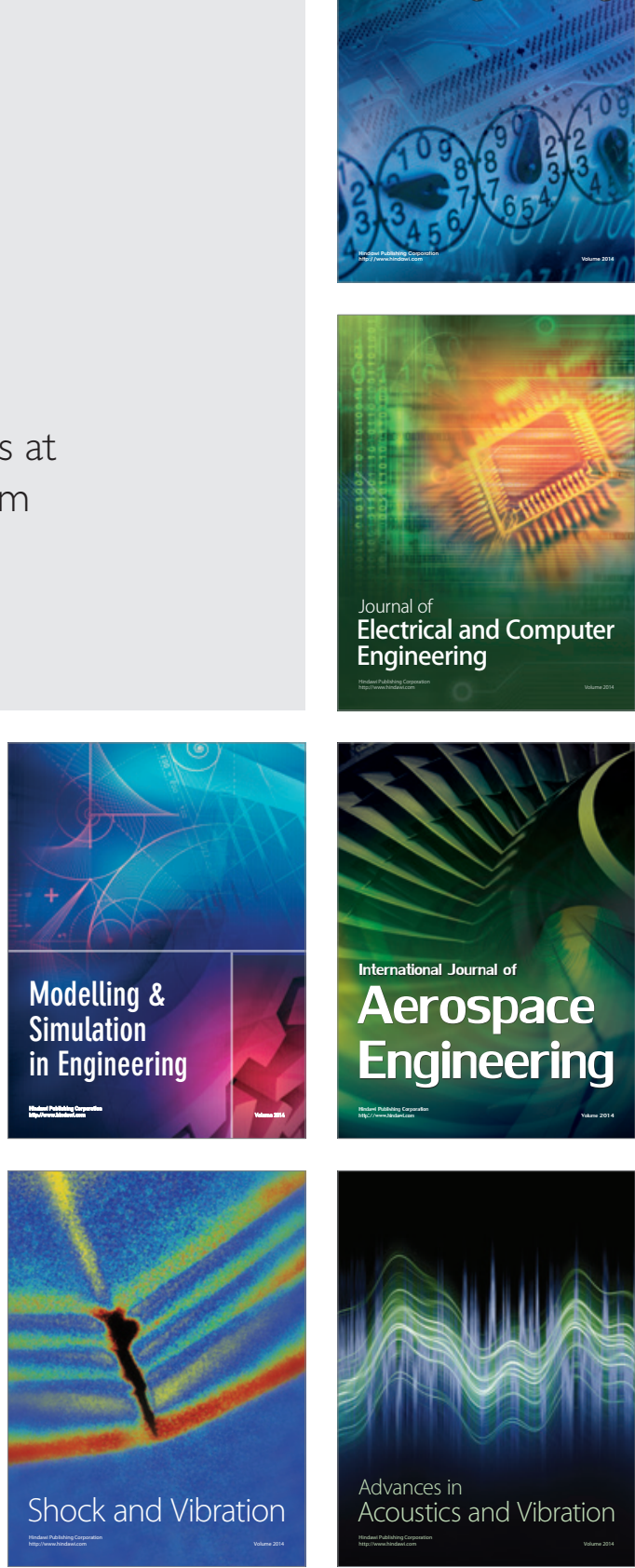